\title{
Transport through an Interacting Quantum Dot Coupled to Two Superconducting Leads
}

\author{
Kicheon Kangt \\ Department of Physics, Korea University, Seoul 136-701, Korea
}

(October 2, 2018)

\begin{abstract}
We derive a formula for the current through an interacting quantum dot coupled to two supercouducting leads, using the non-equilibrium Green's function formalism. It is shown that the formula takes an especially simple form, when the Andreev reflections in the junctions are negligible because of large Coulomb repulsion in the dot. Our formalism provides a new framework to investigate many-body effects of mesoscopic systems in the presence of the superconducting leads. For an example, we describe the resonant tunneling of quasiparticles in the presence of strong Coulomb repulsion in the dot. Further, we discuss the boson assisted transport. Multiple sharp peaks are found in the current-voltage curve, which originate from the singularity of BCS density of states and the boson assisted tunneling.
\end{abstract}

PACS numbers: 72.10.Bg, 73.23.Hk, 73.40.Gk, 74.50.+r

Recent advancement of modern nanofabrication technique has stimulated a lot of interest in the study of electron transport in quantum dot devicest.2. In addition to the Coulomb blockade and the resonant tunneling, a novel non-equilibrium Kondo effect is expected to occur in the low temperature regime for an ultra small quantum dotor artificial atom which is coupled to two metallic leads 3 10. More recently, transport through a nanoparticle is studied experimentally, where the particle is connected to superconducting $(\mathrm{Al})$ leads rather than normal metallic leads 11 . The level spacing of a "superconducting" nanoparticle can be either larger or smaller than its bulk superconducting energy gap, depending on the size of the particle and other parameters such as temperature or external magnetic field. When the level spacing of the nanoparticle is sufficiently large, the superconducting order in the dot disappegrs and the particle can be regarded as an ordinary atom 12 .

In this paper, we present a formulation of calculating the current through an interacting quantum dot with single level coupled to two superconducting leads (Fig. 1). The superconducting leads are characterized by the energy gaps $\Delta_{L}, \Delta_{R}$, and the chemical potentials $\mu_{L}, \mu_{R}$, where $\mu_{L}-\mu_{R}=e V, V$ being the bias voltage across the leads. The subscript $L(R)$ represents the left (right) lead. Using the non-equilibrium Green's function technique, we derive an expression of the steady state current due to quasiparticle processes. It is shown that the current can be expressed in terms of the single-particle spectral density of states of the dot electron, if the Andreev reflection (AR) processes are negligible. In fact, the AR contributions to the current can be neglected when the Coulomb interaction energy $(U)$ in the dot exceeds both the superconducting gaps $\Delta_{L}, \Delta_{R}$ and the coupling between the dot and the leads $\Gamma_{L}, \Gamma_{R}$. It is because, on this condition, the $2 e$-charge fluctuation of the dot is energetically unfavourable. Note that this will be a typical condition of double barrier tunneling structures with suf- ficiently small quantum dot (see e.g.11). In this case the formulation becomes quite simple, because there is no $\mathrm{AC}$ component of the current.

To describe the system, we start with the following model Hamiltonian

$$
\begin{aligned}
\mathcal{H} & =\mathcal{H}_{L}+\mathcal{H}_{R}+\mathcal{H}_{D}\left\{d_{\sigma}^{\dagger} ; d_{\sigma}\right\} \\
& +\sum_{k \sigma \in L, R} V_{k}\left(c_{k \sigma}^{\dagger} d_{\sigma}+d_{\sigma}^{\dagger} c_{k \sigma}\right),
\end{aligned}
$$

where $\mathcal{H}_{L}, \mathcal{H}_{R}$ and $\mathcal{H}_{D}$ represent left, right BCS superconducting leads and the quatum dot, respectively. The last term in the Eq. (1) corresponds to the coupling between the dot and each of the leads. $d_{\sigma}^{\dagger}\left(d_{\sigma}\right)$ creates (destroys) an electron with spin $\sigma$ in the dot, and $c_{k \sigma}^{\dagger}\left(c_{k \sigma}\right)$ creates (destroys) an electron with the momentum $k$ and spin $\sigma$ in either the left $(\mathrm{L})$ or the right $(\mathrm{R})$ lead. In general, $\mathcal{H}_{D}$ includes intra-dot electron-electron interactions, and coupling to an environment like a bosonic bath through the electron-boson interactions 8 .

As noted above, when $U \gg \Delta_{L, R}, \Gamma_{L, R}$ the Andreev reflection processes are prohibited, and DC current flows by the resonant tunneling of quasiparticles. The current flowing out of the lead $\alpha(\alpha=L, R)$ is written by

$$
I_{\alpha}=\frac{e}{\hbar} \sum_{k \sigma \in \alpha} V_{k} \int_{-\infty}^{\infty} \frac{d \omega}{2 \pi}\left(G_{d, k \sigma}^{<}(\omega)-G_{k \sigma, d}^{<}(\omega)\right),
$$

where $G_{d, k \sigma}^{<}(\omega)$ is the Fourier transform of the Keldysh Green's function $G_{d, k \sigma}^{<}(t) \equiv i\left\langle c_{k \sigma}^{\dagger}(0) d_{\sigma}(t)\right\rangle$. By using the Dyson's equation in the Keldysh's formalism, the current due to quasiparticles is given by

$$
\begin{aligned}
I_{\alpha}= & \frac{e}{\hbar} \sum_{k \sigma \in \alpha} V_{k}^{2} \int_{-\infty}^{\infty} \frac{d \omega}{2 \pi}\left[\left(g_{k \sigma}^{<}(\omega)-g_{k \sigma}^{>}(\omega)\right) G_{\sigma}^{<}(\omega)\right. \\
& \left.+g_{k \sigma}^{<}(\omega)\left(G_{\sigma}^{r}(\omega)-G_{\sigma}^{a}(\omega)\right)\right]
\end{aligned}
$$

where $G_{\sigma}^{<}(t) \equiv i\left\langle d_{\sigma}^{\dagger}(0) d_{\sigma}(t)\right\rangle$, and $G_{\sigma}^{r}$ and $G_{\sigma}^{a}$ are the corresponding retarded and advanced Green's functions, 
respectively. The Green's functions denoted by $g$ are the unperturbed Green's functions of the superconducting lead. In deriving the Eq.(3) from the Eq.(2), we have neglected terms containing the off-diagonal Green's functions of dot electron in the Nambu representation because those terms should involve Andreev reflection processes, which is negligible in our case. It should be noted that the "full" Green's functions of the dot represented by $G_{\sigma}$ may include the Andreev reflections, but contribution of those can be neglected when the charging energy in the dot is sufficiently large. Therefore the Eq. (3) can be adopted as the formula for the current in the system.

Using the relations

$$
\begin{aligned}
g_{k \sigma}^{<}(\omega)= & 2 \pi i\left[u_{k}^{2} f_{\alpha}\left(E_{k}\right) \delta\left(\omega-E_{k}\right)\right. \\
& \left.+v_{k}^{2}\left(1-f_{\alpha}\left(E_{k}\right)\right) \delta\left(\omega+E_{k}\right)\right], \\
g_{k \sigma}^{>}(\omega)= & -2 \pi i\left[u_{k}^{2}\left(1-f_{\alpha}\left(E_{k}\right)\right) \delta\left(\omega-E_{k}\right)\right] \\
& \left.+v_{k}^{2} f_{\alpha}\left(E_{k}\right) \delta\left(\omega+E_{k}\right)\right],
\end{aligned}
$$

where $u_{k}^{2}=\frac{1}{2}\left(1+\frac{\epsilon_{k}-\mu_{\alpha}}{E_{k}-\mu_{\alpha}}\right), v_{k}^{2}=\frac{1}{2}\left(1-\frac{\epsilon_{k}-\mu_{\alpha}}{E_{k}-\mu_{\alpha}}\right), \epsilon_{k}$ represents the (normal) single particle energy of the lead, $E_{k}=\mu_{\alpha} \pm \sqrt{\left(\epsilon_{k}-\mu_{\alpha}\right)^{2}+\Delta_{\alpha}^{2}}$, and $f_{\alpha}$ is the Fermi distribution function of the lead $\alpha$, one find

$$
\begin{aligned}
I_{\alpha}= & \frac{2 i e}{h} \sum_{\sigma} \int d \omega \Gamma_{\alpha}^{S}(\omega) \\
& \times\left[f_{\alpha}(\omega)\left(G_{\sigma}^{r}(\omega)-G_{\sigma}^{a}(\omega)\right)+G_{\sigma}^{<}(\omega)\right] .
\end{aligned}
$$

Here $\Gamma_{\alpha}^{S}(\omega)=\Gamma_{\alpha} \zeta_{\alpha}\left(\omega-\mu_{\alpha}\right)$, with $\Gamma_{\alpha}=$ $\pi \sum_{k \in \alpha} V_{k}^{2} \delta\left(\mu_{\alpha}-\epsilon_{k}\right)$ being the constant characterizing coupling between the dot and the lead $\alpha$ which is assumed to be independent of the energy, and $\zeta_{\alpha}(\omega)$ being the dimensionless BCS density of states of the lead $\alpha: \zeta_{\alpha}(\omega)=|\omega| / \sqrt{\omega^{2}-\Delta_{\alpha}^{2}}$ if $|\omega|>\Delta_{\alpha}$, and otherwise 0 . It is straightforward to generallize the Eq. (5) to the system with a quantum dot containing multi-levels. In that case, $\Gamma_{\alpha}^{S}$ and the Green's functions are replaced by $M \times M$ matrices, $M$ being the number of the levels, and then $I_{\alpha}$ is given by the trace of the $M \times M$ matrix in the expression (5). In the steady state, the current is conserved, that is $I_{L}+I_{R}=0$, and thus one can symmetrize the formula for the current as

$$
\begin{aligned}
I= & I_{L}=-I_{R}=\frac{1}{2}\left(I_{L}-I_{R}\right) \\
= & \frac{i e}{h} \sum_{\sigma} \int d \omega\left\{\left(\Gamma_{L}^{S}(\omega) f_{L}(\omega)-\Gamma_{R}(\omega) f_{R}(\omega)\right)\right. \\
& \left.\times\left(G_{\sigma}^{r}(\omega)-G_{\sigma}^{a}(\omega)\right)+\left(\Gamma_{L}^{S}(\omega)-\Gamma_{R}^{S}(\omega)\right) G_{\sigma}^{<}(\omega)\right\}
\end{aligned}
$$

with the condition of the current conservation $\left(I_{L}=\right.$ $\left.-I_{R}\right)$,

$$
\begin{aligned}
& \int d \omega\left(\Gamma_{L}^{S}(\omega)+\Gamma_{R}^{S}(\omega)\right) G_{\sigma}^{<}(\omega) \\
&=-\int d \omega\left(\Gamma_{L}^{S}(\omega) f_{L}(\omega)+\Gamma_{R}^{S}(\omega) f_{R}(\omega)\right) \\
& \times\left(G_{\sigma}^{r}(\omega)-G_{\sigma}^{a}(\omega)\right) .
\end{aligned}
$$

The current formula (6) is reduced to the simple form

$$
I=\frac{2 e}{\hbar} \sum_{\sigma} \int d \omega \tilde{\Gamma}^{S}(\omega)\left\{f_{L}(\omega)-f_{R}(\omega)\right\} \rho_{\sigma}(\omega)
$$

where $\tilde{\Gamma}^{S}(\omega)=\frac{\Gamma_{L}^{S}(\omega) \Gamma_{R}^{S}(\omega)}{\Gamma_{L}^{S}(\omega)+\Gamma_{R}^{S}(\omega)}$ and $\rho_{\sigma}(\omega)=-\frac{1}{\pi} \operatorname{Im} G_{\sigma}^{r}(\omega)$, by using the approximation

$$
\begin{aligned}
& \int d \omega\left(\Gamma_{L}^{S}(\omega)-\Gamma_{R}^{S}(\omega)\right) G_{\sigma}^{<}(\omega) \\
& =-\int d \omega \frac{\Gamma_{L}^{S}(\omega)-\Gamma_{R}^{S}(\omega)}{\Gamma_{L}^{S}(\omega)+\Gamma_{R}^{S}(\omega)} \\
& \times\left(\Gamma_{L}^{S}(\omega) f_{L}(\omega)+\Gamma_{R}^{S}(\omega) f_{R}(\omega)\right)\left(G_{\sigma}^{r}(\omega)-G_{\sigma}^{a}(\omega)\right) .
\end{aligned}
$$

This approximation is made by multiplying the factor $\frac{\Gamma_{L}^{S}(\omega)-\Gamma_{R}^{S}(\omega)}{\Gamma_{L}^{S}(\omega)+\Gamma_{R}^{S}(\omega)}$ on the integrands of both sides of the Eq. (7), and seems to be reasonable since $\frac{\Gamma_{L}^{S}(\omega)-\Gamma_{R}^{S}(\omega)}{\Gamma_{L}^{S}(\omega)+\Gamma_{R}^{S}(\omega)}$ is a smooth function of $\omega$ except for the points $\omega=\mu_{\alpha} \pm \Delta_{\alpha}$ and its absolute value is not larger than one. Further, this approximation becomes exact in the small coupling limit, $\Gamma \ll k_{B} T$, because both $G^{<}$and $G^{r}-G^{a}$ have sharp $\delta$-function-like peaks at resonant level of the dot.

The Eq. (8) is the main result of this paper. Note that the spectral function $\rho_{\sigma}(\omega)$ should be calculated in the presence of the leads, and it includes resonant tunneling, spin flips, inelastic scattering, etc. For the normal metallic leads, $\Gamma_{\alpha}^{S}(\omega)=\Gamma_{\alpha}$, and thys the Eq. (8) is reduced to Meir and Wingreen's formula13. That is, the Eq. (\$) is a generallization of Meir and Wingreen's formula to the case of the superconducting leads.

As an example of the quantum dot, let us consider an Anderson impurity. The Hamiltonian of the dot is

$$
\mathcal{H}_{D}=\sum_{\sigma} \varepsilon_{\sigma} d_{\sigma}^{\dagger} d_{\sigma}+U n_{\uparrow} n_{\downarrow}
$$

where $n_{\sigma}=d_{\sigma}^{\dagger} d_{\sigma}$ is the number operator of the dot electron with spin $\sigma$. If one neglects higher order effects such as the Kondo-like correlations, the Green's function can be simply evaluated by the method of the equation tion and truncation of high order Green's functions 14.14. To begin with, let us introduce $2 \times 2$ Nambu representation of the (retarded) Green's function

$$
\mathbf{G}_{\sigma}(\omega)=\ll \bar{\gamma}_{\sigma}, \bar{\gamma}_{\sigma}^{\dagger} \gg,
$$

where $\bar{\gamma}_{\sigma}=\left(\begin{array}{c}d_{\sigma} \\ d_{-\sigma}^{\dagger}\end{array}\right)$. The equation of motion for $\mathbf{G}_{\sigma}$ yields

$$
\begin{gathered}
\left(\begin{array}{cc}
\omega-\varepsilon_{\sigma} & 0 \\
0 & \omega+\varepsilon_{-\sigma}
\end{array}\right) \mathbf{G}_{\sigma}(\omega) \\
=\mathbf{1}+\sum_{k \in L, R} V_{k} \bar{\sigma}_{z} \ll \bar{\psi}_{k \sigma}, \bar{\gamma}_{\sigma}^{\dagger} \gg \\
+U \bar{\sigma}_{z} \ll \bar{\gamma}_{\sigma}^{(2)}, \bar{\gamma}_{\sigma}^{\dagger} \gg,
\end{gathered}
$$


where $\bar{\sigma}_{z}=\left(\begin{array}{cc}1 & 0 \\ 0 & -1\end{array}\right), \bar{\psi}_{k \sigma}=\left(\begin{array}{c}c_{k \sigma} \\ c_{-k-\sigma}^{\dagger}\end{array}\right), \bar{\gamma}_{\sigma}^{(2)}=$ $\left(\begin{array}{c}n_{-\sigma} d_{\sigma} \\ n_{\sigma} d_{-\sigma}^{\dagger}\end{array}\right)$ and $\mathbf{1}$ is the unit matrix. This equation cannot be solved exactly due to the last term of the R.H.S. In order to get an approximate solution of $\mathbf{G}_{\sigma}$, we consider the equation of motion for the Green's function $\ll \bar{\gamma}_{\sigma}^{(2)}, \bar{\gamma}_{\sigma}^{\dagger} \gg$ and truncate higher order Green's functions which appear on the equation of motion. Adopting this procedure and taking $U \rightarrow \infty$ limit one can find that

$$
G_{\sigma}(\omega)=\left[\mathbf{G}_{\sigma}(\omega)\right]_{11} \approx \frac{1-\left\langle n_{-\sigma}\right\rangle}{\omega-\varepsilon_{\sigma}+i\left(\Gamma_{L}^{S}(\omega)+\Gamma_{R}^{S}(\omega)\right)},
$$

which leads to the resonant-tunneling-like expression of the current

$$
\begin{aligned}
I & =\frac{4 e}{h} \sum_{\sigma}\left(1-\left\langle n_{-\sigma}\right\rangle\right) \int d \omega\left[f_{L}(\omega)-f_{R}(\omega)\right] \\
& \times \frac{\Gamma_{L}^{S}(\omega) \Gamma_{R}^{S}(\omega)}{\left(\omega-\varepsilon_{\sigma}\right)^{2}+\left(\Gamma_{L}^{S}(\omega)+\Gamma_{R}^{S}(\omega)\right)^{2}}
\end{aligned}
$$

The factor $\left(1-\left\langle n_{-\sigma}\right\rangle\right)$ arises from strong Coulomb repulsion. Similar expression to the Eq. (14) has been obtained by Yeyati et al.16, where the dot Hamiltonian is simply simulated by a single non-interacting level, instead of the Eq. (10). Resonance broadening of the spectral function is included in the Eq. (14). The effect of resonance broadeningin the $I-V$ characteristic has been discussed in the Ref.16. It is notable that for a small resonance broadening $\Gamma_{\alpha} \ll \Delta_{\alpha}$, the current via single level directly reflects the BCS singularity in the density of states of the superconducting lead via the function $\Gamma_{\alpha}(\omega)$ (see Fig.2(a)).

While the Eq. (14) is valid for $\Delta_{\alpha} \gg \Gamma_{\alpha}$, Kondolike correlation becomes important on the other limit, $\Delta_{\alpha} \ll \Gamma_{\alpha}$. In order to describe the Kondo effect properly, more elaboratecalculation, such as non-crossing approximation $(\mathrm{NCA}) 17$, is required in calculating the spectral function of the dot electron. For normal metallic leads, NCA has been used to investigate anomalies of the $I-V$ characteristic arising from the Kondo resonances in finite bias voltages 5 . The formalism given in this paper can address the problem of the non-equilibrium Kondo effect in the presence of the superconducting leads.

Another interesting example is the boson-assisted transport. Consider a quantum dot in which the electrons interact with bosonic modes $\omega_{q}$ with the coupling strength $g_{q}$. The Hamiltonian of such a quantum dot is given by

$$
\begin{aligned}
\mathcal{H}_{D} & =\sum_{\sigma} \varepsilon_{\sigma} d_{\sigma}^{\dagger} d_{\sigma}+U n_{\uparrow} n_{\downarrow} \\
& +\sum_{q} \hbar \omega_{q} b_{q}^{\dagger} b_{q}+\hat{n} \sum_{q} g_{q}\left(b_{q}+b_{q}^{\dagger}\right),
\end{aligned}
$$

where $\hat{n}=\sum_{\sigma} n_{\sigma}$ and $b_{q}^{\dagger}\left(b_{q}\right)$ creates (destroys) a boson. For the full Hamiltonian $\mathcal{H}$ of the Eq. (1) with the quantum dot of the Eq. (15), a canonical transformation with $\phi=i \sum_{q}\left(g_{q} / \hbar \omega_{q}\right)\left(\overleftarrow{b_{q}^{\dagger}}-b_{q}\right)$ gives the new effective Hamiltonian 18

$$
\begin{aligned}
\overline{\mathcal{H}} & =e^{-i \hat{n} \phi} \mathcal{H} e^{i \hat{n} \phi} \\
& =\mathcal{H}_{L}+\mathcal{H}_{R}+\overline{\mathcal{H}}_{D}+\overline{\mathcal{H}}_{T},
\end{aligned}
$$

where

$$
\overline{\mathcal{H}}_{D}=\sum_{\sigma} \bar{\varepsilon}_{\sigma} n_{\sigma}+\bar{U} n_{\uparrow} n_{\downarrow}+\sum_{q} \hbar \omega_{q} b_{q}^{\dagger} b_{q}
$$

and

$$
\overline{\mathcal{H}}_{T}=\sum_{k \sigma \in L, R} V_{k}\left(c_{k \sigma}^{\dagger} d_{\sigma} e^{i \phi}+e^{-i \phi} d_{\sigma}^{\dagger} c_{k \sigma}\right) .
$$

The level position and the Coulomb energy in the dot are renormalized by the electron-boson interaction as $\bar{\varepsilon}_{\sigma}=\varepsilon_{\sigma}-\sum_{q} g_{q}^{2} / \hbar \omega_{q}, \bar{U}=U-2 \sum_{q} g_{q}^{2} / \hbar \omega_{q}$. The phase factors $e^{ \pm i \phi}$ which appear in the tunneling term are related to the boson assisted tunneling. With the transformed Hamiltonian $\overline{\mathcal{H}}$, the current formula (8) is modified as

$$
I=\frac{2 e}{\hbar} \sum_{\sigma} \int d \omega \tilde{\Gamma}^{S}(\omega)\left\{f_{L}(\omega)-f_{R}(\omega)\right\} \bar{\rho}_{\sigma}(\omega),
$$

where $\bar{\rho}_{\sigma}$ is the spectral function of the composite particle denoted by $\bar{d}_{\sigma} \equiv d_{\sigma} e^{i \phi}$ :

$$
\bar{\rho}_{\sigma}(\omega)=-\frac{1}{\pi} \operatorname{Im} \ll \bar{d}_{\sigma}, \bar{d}_{\sigma}^{\dagger} \gg .
$$

In general, exact calculation of $\bar{\rho}_{\sigma}(\omega)$ is not possible. However, in the limit of weak coupling between the dot and leads, that is for $\Gamma=\Gamma_{L}+\Gamma_{R} \ll k_{B} T$, the leads can be neglected and the spectral function can be evaluated exactly for that case. We consider the Einstein model, in which all bosons have a same energy $\hbar \omega_{0}$ and are coupled to the electrons in the quantum dot with strength $g_{0}$ In this model the spectral function is given by (see e.g.t8)

$$
\begin{aligned}
\bar{\rho}_{\sigma}(\omega) & =e^{-\alpha\left(2 N_{0}+1\right)} \sum_{l=-\infty}^{\infty} I_{l}\left(2 \alpha\left[N_{0}\left(N_{0}+1\right)\right]^{1 / 2}\right) \\
& \times e^{\beta l \hbar \omega_{0} / 2}\left\{\left(1-\left\langle n_{-\sigma}\right\rangle\right) \delta\left(\omega-\bar{\varepsilon}_{\sigma}-l \hbar \omega_{0}\right)\right. \\
& \left.+\left\langle n_{-\sigma}\right\rangle \delta\left(\omega-\bar{\varepsilon}_{\sigma}-\bar{U}-l \hbar \omega_{0}\right)\right\}
\end{aligned}
$$

where $N_{0}=1 /\left(e^{\beta \hbar \omega_{0}}-1\right)$ and $\alpha=g_{0}^{2} /\left(\hbar \omega_{0}\right)^{2}$ are the boson occupation number and the dimensionless electronboson coupling constant, respectively. The renormalized quantum dot level and the Coulomb repulsion can be written as $\bar{\varepsilon}_{\sigma}=\varepsilon_{\sigma}-\alpha \hbar \omega_{0}, \bar{U}=U-2 \alpha \hbar \omega_{0} . I_{l}(x)$ is the modified Bessel function. Applying the current formula (17) with (18), one can get $I-V$ characteristics in the presence of the electron-boson coupling. 
Fig.2 shows the $I-V$ characteristics for different values of $\alpha$. Here we consider the spin degenerate case $\left(\varepsilon_{\uparrow}=\varepsilon_{\downarrow}=\varepsilon\right)$. In the absence of the electron-boson coupling (Fig.2(a)), the $I-V$ curve consists of two sharp peaks at $e V=2(|\varepsilon|+\Delta)$ and at $e V=2(\varepsilon+U+\Delta)$. These peaks reflect the singularity in the BCS density of states of the leads. This feature, which makes better spectral resolution than the case of the normal metallic leads, have been verified experimentally 11 . Note that there is no thermal broadening in contrast to the case of the normal metallic leads. For normal metallic leads, there are two thermally broadend steps at $e V=2|\varepsilon|, 2(\varepsilon+U)$. For $\alpha \neq 0$, the main peaks are located at $e V=2(|\bar{\varepsilon}|+\Delta), 2(\bar{\varepsilon}+\bar{U}+\Delta)$. Further, we obtain additional side peaks at $e V=2\left(\left|\bar{\varepsilon}+l \hbar \omega_{0}\right|+\Delta\right)$ and at $e V=2\left(\bar{\varepsilon}+\bar{U}+l \hbar \omega_{0}+\Delta\right)$ with positive integer $l$ related to boson emission. Boson absorption processes are negligible because $k_{B} T \ll \hbar \omega_{0}$ is taken here. For weak electron-boson coupling, only single boson emission process contributes to the $I-V$ curve (Fig2.(b)). As the coupling becomes stronger, multiple side peaks appear (Fig2.(c)). For normal metallic leads, side steps arise from the boson assisted tunneling, but those are not clearly shown because of thermal broadening.

In conclusion, we have derived a formula for the current through an interacting quantum dot coupled to two suerconducting leads, when the Andreev reflecction processes are negligible because of the strong Coulomb repulsion in the dot. This formula provides a new framework to study interaction effects in the transport through mesoscopic systems coupled to superconductors. For example, quasiparticle resonant tunneling has been described by an equation of motion method in the limit of large Coulomb repulsion in the dot. Further, current-voltage charactersistics of boson assisted tunneling have been studied. Splitting of BCS singularities, which arises from the boson assisted tunneling, has been shown in the $I-V$ characteristics.

The author thanks B.I. Min for useful discussions. This work has been supported by the Korea Science and Engineering Foundation.
* Electronic address : kckang@silicon.korea.ac.kr

${ }^{1}$ M. A. Kastner, Rev. Mod. Phys. 64, 849 (1992); Phys. Today 46, 24 (1993).

${ }^{2}$ D. C. Ralph and R. A. Buhrman, Phys. Rev. Lett. 72, 3401 (1994).

3 S. Hershfeld, J. H. Davies and J. W. Wilkins, Phys. Rev. Lett. 67, 3720 (1991); Phys. Rev. B 46 (1992).

${ }^{4}$ Y. Meir, N. S. Wingreen and P. A. Lee, Phys. Rev. Lett. 66, 3048 (1991).

${ }^{5}$ Y. Meir, N. S. Wingreen and P. A. Lee, 70, 2601 (1993); N. S. Wingreen and Y. Meir, Phys. Rev. B 49, 11040 (1994).

${ }^{6}$ A. L. Yeyati, A. Martin-Rodeo and F. Flores, Phys. Rev. Lett. 71, 2991 (1993).

7 T. K. Ng, Phys. Rev. Lett. 76, 487 (1996).

${ }^{8}$ C. A. Stafford, Phys. Rev. Lett. 77, 2770 (1996).

${ }^{9}$ J. König, H. Schoeller and G. Schön, Phys. Rev. Lett. 76, 1715 (1996); J. König, J. Schmid, H. Schoeller and G. Schön, Phys. Rev. B 54, 16820 (1996).

${ }^{10}$ K. Kang and B. I. Min, Phys. Rev. B 55, 15412 (1997).

${ }^{11}$ D. C. Ralph, C. T. Black and M. Tinkham, Phys. Rev. Lett. 74, 3241 (1995); C. T. Black, D. C. Ralph and M. Tinkham, Phys. Rev. Lett. 76, 688 (1996); D. C. Ralph, C. T. Black and M. Tinkham, Phys. Rev. Lett. 78, 4087 (1997).

${ }^{12}$ P. W. Anderson, J. Phys. Chem. Solids 11, 28 (1959); J. von Delft, A. D. Jaikin, D. S. Golubev and W. Tichy, Phys. Rev. Lett. 77, 3189 (1996); R. A. Smith and V. Ambegaokar, Phys. Rev. Lett. 77, 4962 (1996); K. A. Matveev and A. I. Larkin, Phys. Rev. Lett. 78, 3749 (1997).

${ }^{13}$ Y. Meir and N. S. Wingreen, Phys. Rev. Lett. 68, 2512 (1992).

${ }^{14}$ G. Czycholl, Phys. Rep. 143, 277 (1986).

${ }^{15}$ K. Kang and B. I. Min, Phys. Rev. B 52, 10689 (1995).

${ }^{16}$ A. L. Yeyati, J. C. Cuevas, A. López-Dávalos and A. Martín-Rodero, Phys. Rev. B 55, R 6137 (1997).

${ }^{17}$ N. E. Bickers, Rev. Mod. Phys. 59, 845 (1987).

${ }^{18}$ G. D. Mahan, Many-Particle Physics, 2nd ed. (Plenum, New York 1990).

FIG. 1. Schematic diagram of a quantum dot connected to two superconducting leads. The superconducting leads are characterized by the chemical potentials $\mu_{L}, \mu_{R}$ and the energy gaps $\Delta_{L}, \Delta_{R}$. An eletron current $I$ flows from left to right if $\mu_{L}-\mu_{R}=e V>0$.

FIG. 2. Current-voltage characteristics (a) without, and (b)(c) with boson assisted tunneling. Here $\Delta_{L}=\Delta_{R}=\Delta_{\text {, }}$ $\Gamma_{L}=\Gamma_{R}=\Gamma / 2$ and $\mu_{L}=e V / 2, \mu_{R}=-e V / 2$ are considered. Other parameters used here are $k_{B} T=0.1, \varepsilon=\varepsilon_{\uparrow}=\varepsilon_{\downarrow}=-2$, $U=8, \hbar \omega_{0}=0.6$ in the unit of $\Delta$. Full lines represent the results for the superconducting leads, while dashed lines being corresponding results for the normal metallic leads. 


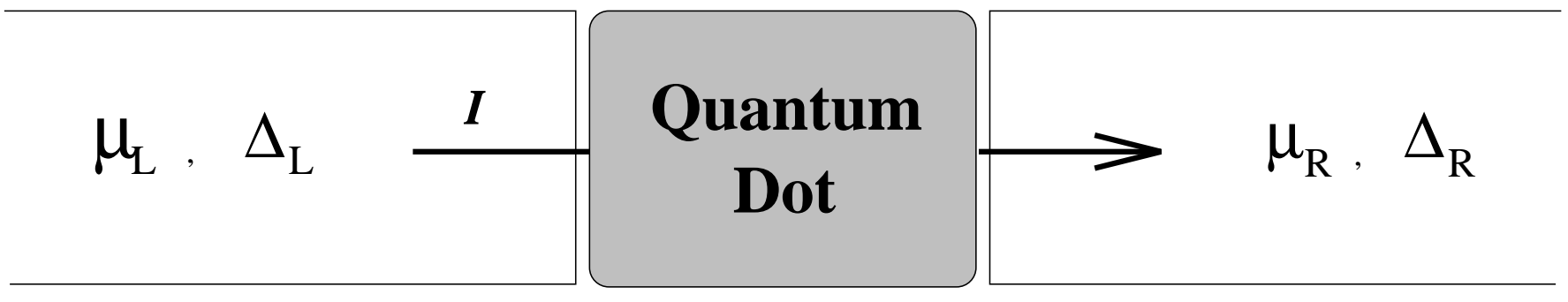




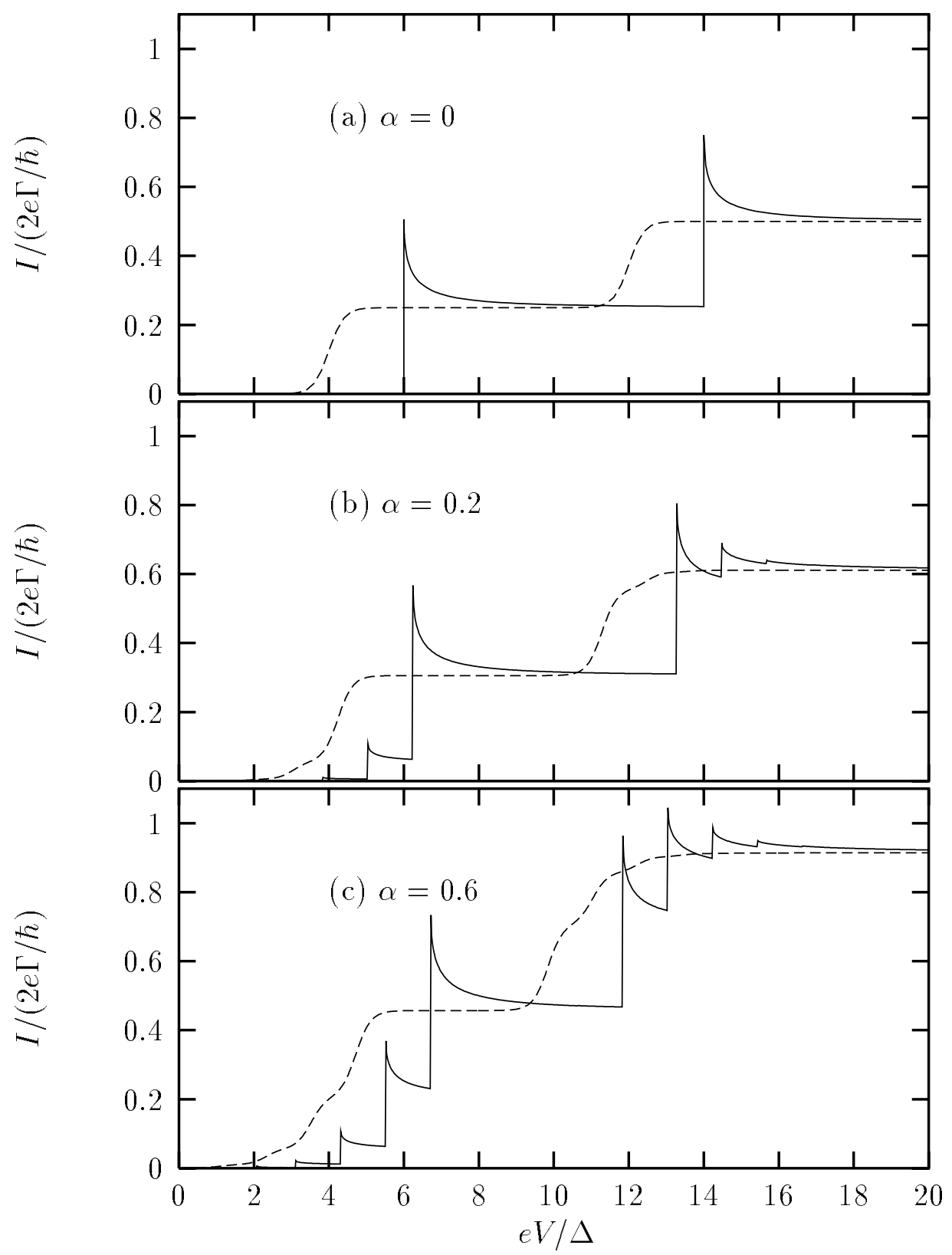

Fig.2 